\title{
Unconditional convergence of difference equations
}

Daniel Franco and Juan Peran*

"Correspondence:

jperan@ind.uned.es

Departamento de Matemática

Aplicada, Universidad Nacional de

Educación a Distancia (UNED), C/

Juan del Rosal 12, Madrid, 28040,

Spain

\begin{abstract}
We put forward the notion of unconditional convergence to an equilibrium of a difference equation. Roughly speaking, it means that can be constructed a wide family of higher order difference equations, which inherit the asymptotic behavior of the original difference equation. We present a sufficient condition for guaranteeing that a second-order difference equation possesses an unconditional stable attractor. Finally, we show how our results can be applied to two families of difference equations recently considered in the literature.
\end{abstract}

MSC: 39A11

Keywords: difference equations; global asymptotic stability

\section{Introduction}

It is somewhat frequent that the global asymptotic stability of a family of difference equations can be extended to some higher-order ones (see, for example, [1-4]). Consider the following simple example. If $\varphi$ is the map $\varphi(x, y)=1+(a x / y)$, the sequence $y_{n}$ defined by $y_{n}=\varphi\left(x, y_{n-1}\right)$, that is,

$$
y_{n}=1+\frac{a x}{y_{n-1}}
$$

with $y_{1}, a, x>0$, converges to $F_{\varphi}(x)=(1+\sqrt{1+4 a x}) / 2$ for any $y_{1}$. Observe that $F_{\varphi}$ is the function satisfying $\varphi\left(x, F_{\varphi}(x)\right)=F_{\varphi}(x)$. Obviously, the second-order difference equation

$$
y_{n}=1+\frac{a x}{y_{n-2}}
$$

also converges to $F_{\varphi}(x)$ for any $y_{1}, y_{2}, a, x>0$. Let us continue to add complexity, by considering the second-order difference equations

$$
\begin{aligned}
& y_{n}=1+\frac{a y_{n-1}}{y_{n-2}}, \\
& y_{n}=1+\frac{a y_{n-2}}{y_{n-1}} .
\end{aligned}
$$

For all $y_{1}, y_{2}, a>0$, the sequence defined by Equation (1) converges to the unique fixed point $\mu_{\varphi}=a+1$ of the function $F_{\varphi}$. However, the behavior of Equation (2) depends on the parameter $a$ :

(0) 2013 Franco and Peran; licensee Springer. This is an Open Access article distributed under the terms of the Creative Commons Attribution License (http://creativecommons.org/licenses/by/2.0), which permits unrestricted use, distribution, and reproduction in any medium, provided the original work is properly cited. 
- For $a \geq 1$, the odd and even index terms converge respectively to some limits, $\mu_{1} \in[1,+\infty]$ and $\mu_{1} /\left(\mu_{1}-1\right) \cap[1,+\infty]$, where $\mu_{1}$ may depend on $y_{1}, y_{2}$ (for $a=1$ ).

- For $0<a<1$, it converges to $\mu_{\varphi}=a+1$, whatever the choice of $y_{1}, y_{2}>0$ one makes. No sophisticated tools are needed to reach those conclusions: It suffices to note that the set

$$
A=\left\{n:\left(y_{n+3}-y_{n+1}\right)\left(y_{n+2}-y_{n}\right) \geq 0\right\}
$$

must be either finite or equal to $\mathbb{N}$. As the sequences $y_{2 n+1}$ and $y_{2 n}$ are then both eventually monotone, they converge in $[1,+\infty]$ to some limits, say $\mu_{1}$ and $\mu_{2}$, satisfying

$$
\mu_{1}=1+\frac{a \mu_{1}}{\mu_{2}} \quad \text { and } \quad \mu_{2}=1+\frac{a \mu_{2}}{\mu_{1}} .
$$

Therefore, one of the following statements holds: $\mu_{2}=\mu_{1} /\left(\mu_{1}-1\right) \cap[1,+\infty]$, with $a=1$, or $\left\{\mu_{1}\right\} \cup\left\{\mu_{2}\right\} \in\{\{1,+\infty\},\{1+a\}\}$.

If $\left\{\mu_{1}\right\} \cup\left\{\mu_{2}\right\}=\{1,+\infty\}$, then that of the sequences, $y_{2 n+1}$ or $y_{2 n}$, which converges to $+\infty$, has to be nondecreasing. Just look at Equation (2) to conclude that $a \geq 1$ whenever $\left\{\mu_{1}\right\} \cup\left\{\mu_{2}\right\}=\{1,+\infty\}$.

The case we are interested in is $0<a<1$ and we will say that $\mu_{\varphi}=1+a$ is an unconditional attractor for the map $\varphi$, that is, we would consider $\varphi(x, y)=1+(a x / y)$ with $0<a<1$ to observe that, not only (1) and (2), but all the following recursive sequences converge to $\mu_{\varphi}=1+a$, whatever the choice of $y_{1}, \ldots, y_{\max \{k, m\}}>0$ we make:

$$
\begin{aligned}
& y_{n}=1+\frac{a y_{n-k}}{y_{n-m}}, \\
& y_{n}=1+a \frac{y_{n-k+1}+y_{n-k}}{y_{n-m+1}+y_{n-m}}, \\
& y_{n}=1+a \sqrt{\frac{y_{n-k+1} y_{n-k}}{y_{n-m+1} y_{n-m}}}, \\
& y_{n}=1+a \frac{\max \left\{y_{n-k+1}, y_{n-k}\right\}}{\min \left\{y_{n-m+1}, y_{n-m}\right\}},
\end{aligned}
$$

In this paper, we proceed as follows. The next section is dedicated to notation and a technical result of independent interest. In Section 3, we introduce the main definition and the main result in this paper, unconditional convergence and a sufficient condition for having it in a general framework. We conclude, in Section 4, showing how the later theorem can be applied to provide short proofs for some recent convergence results on two families of difference equations and to improve them.

\section{Preliminaries}

This section is mainly devoted to the notation we employ. In the first part, we establish some operations between subsets of real numbers and we clarify how we identify a function with a multifunction. We noticed that set-valued difference equations are not concerned with us in this paper. The reason for dealing with those set operations and notation is because it allows us to manage unboundedness and singular situations in a homogeneous way. In the second part, we introduce the families of maps $\Lambda_{m}^{k}$ (a kind of averages 
of their variables) that we shall employ in the definition of unconditional convergence. We finish the section with a technical result on monotone sequences converging to the fixed point of a monotone continuous function.

\subsection{Basic notations}

We consider the two points compactification $\overline{\mathbb{R}}=[-\infty,+\infty]$ of $\mathbb{R}$ endowed with the usual order and compact topology.

\subsubsection{Operations and preorder in $2^{\overline{\mathbb{R}}} \backslash\{\emptyset\}$}

We define the operations '+,' '-, ' ' ' and 'l' in $2^{\overline{\mathbb{R}}} \backslash\{\emptyset\}$ by

$$
A * B=\left\{\limsup \left(a_{n} * b_{n}\right): a_{n}, b_{n}, a_{n} * b_{n} \in \mathbb{R} \text { for all } n \in \mathbb{N} \text { and } \lim a_{n} \in A, \lim b_{n} \in B\right\},
$$

where $*$ stands for '+,' '-,' '.' or ' $\%$ '. We also agree to write $A * \emptyset=\emptyset * A=\emptyset$.

Remark 1 We introduce the above notation in order to manage unboundedness and singular situations, but we point out that these are natural set-valued extensions for the arithmetic operations. Let $X, Y$ be compact (Hausdorff) spaces, $U$ a dense subset of $X$ and $f: U \rightarrow Y$. The closure $\overline{\operatorname{Gr}(f)}$ of the graph of $f$ in $X \times Y$ defines an upper semicontinuous compact-valued map $\bar{f}: X \rightarrow 2^{Y}$ by $\bar{f}(x)=\{y \in Y:(x, y) \in \overline{\operatorname{Gr}(f)}\}$, that is, by $\operatorname{Gr}(\bar{f})=\overline{\operatorname{Gr}(f)}$ (see [5]). Furthermore, as usual, one writes $\bar{f}(A)=\bigcup_{x \in A} \bar{f}(x)$ for $A \in 2^{X}$, thereby obtaining a map $\bar{f}: 2^{X} \rightarrow 2^{Y}$.

To extend arithmetic operations, consider $X=\overline{\mathbb{R}} \times \overline{\mathbb{R}}, Y=\overline{\mathbb{R}}$ and $U=\mathbb{R} \times \mathbb{R}$, when $f$ denotes addition, substraction or multiplication, and $U=\mathbb{R} \times(\mathbb{R} \backslash\{0\})$, when $f$ denotes division.

Also define $A \leq B$ (respectively $A<B$ ) to be true if and only if $A \neq \emptyset, B \neq \emptyset$ and $a \leq b$ (respectively $a<b$ ) for all $a \in A, b \in B$. Here $A, B \in 2^{\overline{\mathbb{R}}}$.

Notice that both relations $\leq$ and $<$ are transitive but neither reflexive nor symmetric.

\subsubsection{Canonical injections}

When no confusion is likely to arise, we identify $a \in \overline{\mathbb{R}}$ with $\{a\} \in 2^{\overline{\mathbb{R}}}$, that is, in the sequel we consider the fixed injection $a \rightarrow\{a\}$ of $\overline{\mathbb{R}}$ into $2^{\overline{\mathbb{R}}}$ and we identify $\overline{\mathbb{R}}$ with its image. We must point out that, under this convention, when $a$ is expected to be subset of $A$, we understand ' $a \in A$ ' as 'there is $b \in A$ with $a=\{b\}$. For instance, one has $0 \cdot(+\infty)=\overline{\mathbb{R}}$, $1 / 0=\{-\infty,+\infty\}$

\subsubsection{Extension of a function as a multifunction}

Consider a map $h: \overline{\mathbb{R}}^{m} \rightarrow 2^{\overline{\mathbb{R}}}$ and denote by $\mathcal{D}(h)$ the set formed by those $x \in \overline{\mathbb{R}}^{m}$ for which there is $b \in \overline{\mathbb{R}}$ with $h(x)=\{b\}$.

If $A \in 2^{\left(\overline{\mathbb{R}}^{m}\right)}$, then $h(A) \in 2^{\overline{\mathbb{R}}}$ is defined to be $\bigcup_{a \in A} h(a)$. Also, if $B \in\left(2^{\overline{\mathbb{R}}}\right)^{m}$, then $h(B) \in 2^{\overline{\mathbb{R}}}$ is defined to be $h(B)=h\left(B_{1} \times \cdots \times B_{m}\right)$.

For each function $\varphi: U \subset \overline{\mathbb{R}}^{m} \rightarrow \overline{\mathbb{R}}$, let $\widehat{\varphi}: \overline{\mathbb{R}}^{m} \rightarrow 2^{\overline{\mathbb{R}}}$ be defined by

$$
\widehat{\varphi}(x)=\left\{\lim \sup \varphi\left(x_{n}\right): x_{n} \in U \text { for all } n \in \mathbb{N} \text { and } \lim x_{n}=x\right\}
$$


It is obvious that $\widehat{\varphi}(x) \neq \emptyset$ if and only if $x$ is in the closure $\bar{U}$ of $U$ in $\overline{\mathbb{R}}^{m}$. Also notice that

$$
U \subset \mathcal{D}(\widehat{\varphi}) \subset \bar{U}
$$

when $\varphi$ is continuous. In this case, and if no confusion is likely to arise, we agree to denote also by $\varphi$ the map $\widehat{\varphi}$. For example, we write

$$
\varphi(0)=[-1,1] ; \quad \varphi(-\infty)=\varphi(+\infty)=0 ; \quad \mathcal{D}(\varphi)=\overline{\mathbb{R}} \backslash\{0\}
$$

when $U=\mathbb{R} \backslash\{0\}$ and $\varphi(x)=\sin (1 / x)$.

\subsection{The maps in $\Lambda_{m}^{k}$ and $\Lambda^{k}$}

As we have announced, the unconditional convergence of a difference equation guarantees that there exists a family of difference equations that inherit its asymptotic behavior. Here, we define the set of functions that we employ to construct that family of difference equations.

For $k, m \in \mathbb{N}$, let $\Lambda_{m}^{k}$ be the set formed by the maps $\lambda: \overline{\mathbb{R}}^{m} \rightarrow \overline{\mathbb{R}}^{k}$ such that

$$
\min _{1 \leq j \leq m} x_{j} \leq \lambda_{i}(x) \leq \max _{1 \leq j \leq m} x_{j} \quad \text { for all } x \in \overline{\mathbb{R}}^{m}, 1 \leq i \leq k .
$$

Notice that $\lambda \circ \gamma \in \Lambda_{m}^{k}$ whenever $\lambda \in \Lambda_{r}^{k}, \gamma \in \Lambda_{m}^{r}$. Let $\Lambda^{k}$ be defined as follows:

$$
\Lambda^{k}=\bigcup_{m \in \mathbb{N}} \Lambda_{m}^{k}
$$

We note that the functions in $\Lambda^{k}$ satisfy that their behavior is enveloped by the maximum and minimum functions of its variables, which is a common hypothesis in studying higher order nonlinear difference equations.

Some trivial examples of functions in $\Lambda^{1}$ are:

- $\lambda\left(x_{1}, \ldots, x_{m}\right)=\sum_{j=1}^{m} \alpha_{j} x_{j}$, with $\alpha_{j} \geq 0$ for $j=1, \ldots, m, \sum_{j=1}^{m} \alpha_{j}=1$.

An important particular case is $\alpha_{j_{0}}=1, \alpha_{j}=0$ for $j \neq j_{0}$.

- $\lambda\left(x_{1}, \ldots, x_{m}\right)=\left\{\begin{array}{ll}\prod_{j=1}^{m} x_{j} & \text { if } \min _{1 \leq j \leq m} x_{j}>0, \\ \min _{1 \leq j \leq m} x_{j} & \text { if } \min _{1 \leq j \leq m} x_{j} \leq 0,\end{array}\right.$ with $\alpha_{j} \geq 0$ for $j=1, \ldots, m, \sum_{j=1}^{m} \alpha_{j}=1$.

We refer to this function simply as $\lambda\left(x_{1}, \ldots, x_{m}\right)=\prod_{j=1}^{m} x_{j}^{\alpha_{j}}$, when it is assumed that $\lambda \in \Lambda^{1}$.

- $\lambda\left(x_{1}, \ldots, x_{m}\right)=\max _{j \in J} x_{j}$, where $J$ is a nonempty subset of $\{1, \ldots, m\}$.

- $\lambda\left(x_{1}, \ldots, x_{m}\right)=\min _{j \in J} x_{j}$, where $J$ is a nonempty subset of $\{1, \ldots, m\}$.

\subsection{A technical result}

Assume $-\infty \leq a<b \leq+\infty$, in the rest of this section. Recall that a continuous nonincreasing function $F:[a, b] \rightarrow[a, b]$ has a unique fixed point $\mu \in[a, b]$, that is, $\{\mu\}=$ $\operatorname{Fix}(F)$.

Lemma 1 Let $F:[a, b] \rightarrow[a, b]$ be a continuous non-increasing function, $\{\mu\}=\operatorname{Fix}(F)$ and $\epsilon>0$. Define $F(x)=F(a)$ for $x<a, F(x)=F(b)$ for $x>b$ and

$$
a_{0}=a ; \quad b_{0}=F\left(a_{0}\right) ; \quad a_{k}=F\left(b_{k-1}+\frac{\epsilon}{k}\right) ; \quad b_{k}=F\left(a_{k}-\frac{\epsilon}{k}\right)
$$

for $k \geq 1$. 
Then $\left(a_{k}\right)$ and $\left(b_{k}\right)$ are, respectively, a nondecreasing and a nonincreasing sequence in $[a, b]$. Furthermore, $a_{k} \leq \mu \leq b_{k}$ for all $k$ and $\left\{\lim a_{k}, \mu, \lim b_{k}\right\} \subset \operatorname{Fix}(F \circ F)$.

Proof Since the map $F$ is nonincreasing and taking into account the hypothesis $a_{0} \leq a_{1}$, we see that $\left(a_{k}\right)$ is a nondecreasing sequence. Assume $a_{k-1} \leq a_{k}$ and $a_{k}>a_{k+1}$ to reach a contradiction

$$
\begin{aligned}
a_{k}>a_{k+1} & \Rightarrow F\left(b_{k-1}+\frac{\epsilon}{k}\right)>F\left(b_{k}+\frac{\epsilon}{k+1}\right) \\
& \Rightarrow b_{k-1}+\frac{\epsilon}{k}<b_{k}+\frac{\epsilon}{k+1} \Rightarrow b_{k-1}<b_{k} \\
& \Rightarrow F\left(a_{k-1}-\frac{\epsilon}{k-1}\right)<F\left(a_{k}-\frac{\epsilon}{k}\right) \\
& \Rightarrow a_{k-1}-\frac{\epsilon}{k-1}>a_{k}-\frac{\epsilon}{k} \Rightarrow a_{k-1}>a_{k} .
\end{aligned}
$$

Therefore, $\left(a_{k}\right)$ is a nondecreasing sequence, so by definition, $\left(b_{k}\right)$ is nonincreasing.

On the other hand, as $b_{0}=F\left(a_{0}\right) \geq F(\mu)=\mu \geq a_{0}$, we see by induction that $a_{k} \leq \mu \leq b_{k}$ for all $k$,

$$
a_{k}=F\left(b_{k-1}+\frac{\epsilon}{k}\right) \leq F(\mu)=\mu=F(\mu) \leq F\left(a_{k}-\frac{\epsilon}{k}\right)=b_{k} \quad \text { for } k \geq 1
$$

Because of the continuity of $F$, we conclude that

$$
\lim a_{k}=F\left(\lim b_{k}\right)=F\left(F\left(\lim a_{k}\right)\right)
$$

and

$$
\lim b_{k}=F\left(\lim a_{k}\right)=F\left(F\left(\lim b_{k}\right)\right) .
$$

Remark 2 Suppose $F$ not to be identically equal to $+\infty$ and let $x \in[a, \mu)$. The map

$$
\epsilon \rightarrow F(F(x)+\epsilon)-\epsilon
$$

is decreasing in the set

$$
\{\epsilon \geq 0: F(F(x)+\epsilon)<+\infty\}
$$

Unless $F(x)=b<+\infty$, the map $F$ verifies $F(F(x))>x$ if and only if there exists $\epsilon_{0}>0$ such that $F(F(x)+\epsilon)-\epsilon>x$ for all $\epsilon \in\left[0, \epsilon_{0}\right)$.

Therefore, if $F(F(a))>a$, there exists $\epsilon>0$ such that $F(F(a)+\epsilon)-\epsilon \geq a$ and taking $a=a_{0}$

$$
a \leq F(F(a)+\epsilon)-\epsilon=a_{1}-\epsilon \leq a_{k}-\frac{\epsilon}{k} \leq \mu \leq b_{k-1}+\frac{\epsilon}{k} \leq b_{0}+\epsilon=F(a)+\epsilon \leq b
$$

As a consequence, $\left(a_{k}\right),\left(b_{k}\right)$ are well defined, without the need of extending $F$. 


\section{Unconditional convergence to a point}

For a map $h: \overline{\mathbb{R}}^{k} \rightarrow 2^{\overline{\mathbb{R}}}$, the difference equation

$$
y_{n}=h\left(y_{n-1}, \ldots, y_{n-k}\right)
$$

is always well defined whatever the initial points $y_{1}, \ldots, y_{k} \in \overline{\mathbb{R}}$ are, even though the $y_{n}$ are subsets of $\overline{\mathbb{R}}$, rather than points.

A point $\mu \in \overline{\mathbb{R}}$ is said to be an equilibrium for the map $h$ if $h(\mu, \ldots, \mu)=\{\mu\}$. The equilibrium $\mu$ is said to be stable if, for each neighborhood $V$ of $\mu$ in $\overline{\mathbb{R}}$, there is a neighborhood $W$ of $(\mu, \ldots, \mu)$ in $\mathcal{D}(h)$ such that $y_{n} \in V$ for all $n$, whenever $\left(y_{1}, \ldots, y_{k}\right) \in W$.

The equilibrium $\mu$ is said to be an attractor in a neighborhood $V$ of $\mu$ in $\overline{\mathbb{R}}$, if $y_{n} \in \overline{\mathbb{R}}$ for all $n$ and $y_{n} \rightarrow \mu$ in $\overline{\mathbb{R}}$, whenever $y_{n} \in V$ for $n \leq k$.

Definition 1 The point $\mu$ is said to be an unconditional equilibrium of $h$ (respectively, unconditional stable equilibrium, unconditional attractor in $V$ ) if it is an equilibrium (respectively, stable equilibrium, attractor in $V$ ) of $h \circ \lambda$ for all $\lambda \in \Lambda^{k}$.

Definition 2 We define the equilibria, stable equilibria, attractors, unconditional equilibria, unconditional stable equilibria and unconditional attractors of a continuous function $\varphi: U \subset \overline{\mathbb{R}}^{k} \rightarrow \overline{\mathbb{R}}$ to be those of $\widehat{\varphi}$.

\subsection{Sufficient condition for unconditional convergence}

After giving Definitions 1 and 2 we are going to prove a result guaranteeing that a general second order difference equation as in (4) has an unconditional stable attractor.

Let $-\infty<c \leq a<b \leq d \leq+\infty$ and consider in the sequel a continuous function $\varphi:(a, b) \times(c, d) \rightarrow(c, d)$, satisfying the following conditions:

(H1) $\varphi\left(x_{1}, y\right)<\varphi\left(x_{2}, y\right)$, whenever $a<x_{2}<x_{1}<b$ and $c<y<d$.

(H2) There exists $F_{\varphi}:[a, b] \rightarrow[a, b]$ such that

$$
\frac{F_{\varphi}(x)-y}{\varphi(x, y)-y} \geq 1
$$

whenever $y \in(c, d) \backslash\left\{F_{\varphi}(x)\right\}$.

The functions $\varphi(\cdot, y):[a, b] \rightarrow[c, d]$ and $\varphi(x, \cdot):(c, d) \rightarrow(c, d)$ are defined in the obvious way. Notice that $\varphi(a, \cdot)$ is the limit of a monotone increasing sequence of continuous functions, thus it is lower-semicontinuous, likewise $\varphi(b, \cdot)$ is an upper-semicontinuous function. Remember that we denote both $\varphi$ and $\widehat{\varphi}$ by $\varphi$.

The next lemma, which we prove at the end of this section, shows that if $(\mathrm{H} 1)$ and $(\mathrm{H} 2)$ holds we can get some information about the behavior and properties of $\varphi$ and $F_{\varphi}$.

Lemma 2 Let $\varphi:(a, b) \times(c, d) \rightarrow(c, d)$, where $-\infty<c \leq a<b \leq d \leq+\infty$, be a continuous function satisfying (H1) and (H2). Then the function $F_{\varphi}$ in $(\mathrm{H} 2)$ is unique and it is a continuous nonincreasing map, thus it has a unique fixed point $\mu_{\varphi}$. Furthermore,

(i) $a<\varphi(x, y)<b$ for all $x, y \in(a, b)$ and $a \leq \varphi(x, y) \leq b$ for all $x, y \in[a, b]$.

(ii) If $x \in[a, b], y \in(c, d)$ and $\varphi(x, y)=y$, then $y=F_{\varphi}(x)$.

(iii) $\varphi\left(x, F_{\varphi}(x)\right)=F_{\varphi}(x)$ for all $x \in[a, b]$.

(iv) $F_{\varphi}$ is decreasing in $F_{\varphi}^{-1}((a, b))$. 
We are in conditions of presenting and proving our main result.

Theorem 1 Let $\varphi:(a, b) \times(c, d) \rightarrow(c, d)$, where $-\infty<c \leq a<b \leq d \leq+\infty$, be a continuous function satisfying $(\mathrm{H} 1)$ and $(\mathrm{H} 2)$. If $\mu_{\varphi} \in(a, b)$ and $\operatorname{Fix}\left(F_{\varphi} \circ F_{\varphi}\right)=\operatorname{Fix}\left(F_{\varphi}\right)$, then $\mu_{\varphi}$ is an unconditional stable attractor of $\varphi$ in $(a, b)$.

Proof of Theorem 1 Consider $\lambda \in \Lambda_{m}^{2}$ and denote

$$
y_{n}=\varphi \circ \lambda\left(y_{n-1}, \ldots, y_{n-m}\right)
$$

for some $y_{1}, \ldots, y_{m} \in(a, b)$. Notice that $y_{n} \in(a, b)$ for all $n$, as a consequence of (i) in Lemma 2.

We are going to prove first that $\mu_{\varphi}$ is a stable equilibrium of $\varphi \circ \lambda$. By (iii) in Lemma 2 , as

$$
\lambda\left(\mu_{\varphi}, \ldots, \mu_{\varphi}\right)=\left(\mu_{\varphi}, \mu_{\varphi}\right),
$$

we see that $\mu_{\varphi}$ is an equilibrium.

Let $\epsilon \in\left(0, \min \left\{\mu_{\varphi}-a, b-\mu_{\varphi}\right\}\right)$. Because of the continuity of $F_{\varphi}$, there is $a^{\prime} \in\left(\mu_{\varphi}-\epsilon, \mu_{\varphi}\right)$ such that

$$
b^{\prime} \equiv F_{\varphi}\left(a^{\prime}\right) \in\left(\mu_{\varphi}, \mu_{\varphi}+\epsilon\right) .
$$

As $\operatorname{Fix}\left(F_{\varphi} \circ F_{\varphi}\right)=\operatorname{Fix}\left(F_{\varphi}\right)$ and $F_{\varphi}\left(F_{\varphi}(a)\right) \geq a$, we have

$$
F_{\varphi}\left(F_{\varphi}(x)\right)>x \quad \text { for all } x \in\left[a, \mu_{\varphi}\right) .
$$

If $x \in\left[a^{\prime}, b^{\prime}\right]$, then

$$
F_{\varphi}(x) \leq F_{\varphi}\left(a^{\prime}\right)=b^{\prime}
$$

and

$$
F_{\varphi}(x) \geq F_{\varphi}\left(b^{\prime}\right)=F_{\varphi}\left(F_{\varphi}\left(a^{\prime}\right)\right)>a^{\prime} .
$$

Therefore, $F_{\varphi}\left(\left[a^{\prime}, b^{\prime}\right]\right) \subset\left[a^{\prime}, b^{\prime}\right]$.

By replacing $a, b$ by $a^{\prime}, b^{\prime}$ in Lemma $2(\mathrm{i})$, we see that

$$
y_{n} \in\left(a^{\prime}, b^{\prime}\right) \subset\left(\mu_{\varphi}-\epsilon, \mu_{\varphi}+\epsilon\right) \text { for all } n,
$$

whenever $y_{n} \in\left(a^{\prime}, b^{\prime}\right)$ for $n \leq m$, thus $\mu_{\varphi}$ is an unconditional stable equilibrium of $\varphi$.

Now, if we see that

$$
\lim F_{\varphi}\left(y_{n}\right)=\mu_{\varphi},
$$


we are done with the whole proof. Indeed, for each accumulation point $\bar{y}$ of $\left(y_{n}\right)$, one would have

$$
F_{\varphi}\left(\mu_{\varphi}\right)=\mu_{\varphi}=F_{\varphi}(\bar{y})
$$

because of the continuity of $F_{\varphi}$. As $\mu_{\varphi} \in(a, b)$, this implies $\bar{y}=\mu_{\varphi}$.

Therefore, as a consequence of Lemma 1, it suffices to find an increasing sequence $n_{k}$ of natural numbers such that

$$
a_{k} \leq F_{\varphi}\left(y_{n}\right) \leq b_{k} \text { for all } k \geq 0, n \geq n_{k} \text {. }
$$

Here, $a_{k}$ and $b_{k}$ are defined as in Lemma 1 , with $a_{0}=a$ and $\epsilon=1$,

$$
b_{0}=F_{\varphi}(a) ; \quad a_{k}=F_{\varphi}\left(b_{k-1}+\frac{1}{k}\right) ; \quad b_{k}=F_{\varphi}\left(a_{k}-\frac{1}{k}\right) \quad \text { for } k \geq 1
$$

Let $n_{0}=m$, so that

$$
a_{0} \leq F_{\varphi}\left(y_{n}\right) \leq F_{\varphi}(a)=b_{0} \text { for } n \geq n_{0} .
$$

Having in mind that $\lambda \in \Lambda_{m}^{2}$ satisfies (3), we find $n_{k}$ from $n_{k-1}$ as follows. Denote

$$
z_{k}=F_{\varphi}^{-1}\left(b_{k-1}\right)
$$

and momentarily assume $n>n_{k-1}+m$ and $b_{k-1}<y_{n}$ in such a way that

$$
\begin{aligned}
b_{k-1} & <y_{n}=\varphi\left(\lambda_{1}\left(y_{n-1}, \ldots, y_{n-m}\right), \lambda_{2}\left(y_{n-1}, \ldots, y_{n-m}\right)\right) \\
& \leq \varphi\left(\min \left\{y_{n-1}, \ldots, y_{n-m}\right\}, \lambda_{2}\left(y_{n-1}, \ldots, y_{n-m}\right)\right) \\
& \leq \varphi\left(z_{k}, \lambda_{2}\left(y_{n-1}, \ldots, y_{n-m}\right)\right),
\end{aligned}
$$

which implies

$$
\varphi\left(z_{k}, \lambda_{2}\left(y_{n-1}, \ldots, y_{n-m}\right)\right) \leq \lambda_{2}\left(y_{n-1}, \ldots, y_{n-m}\right)
$$

and then

$$
y_{n} \leq \max \left\{b_{k-1}, \lambda_{2}\left(y_{n-1}, \ldots, y_{n-m}\right)\right\} \leq \max \left\{b_{k-1}, y_{n-1}, \ldots, y_{n-m}\right\} \equiv w_{n}
$$

for all $n>n_{k-1}+m$.

As a consequence, the nonincreasing sequence $w_{n}$ is bounded below by $b_{k-1}$. It cannot be the case that $\lim w_{n}>b_{k-1}$, because in such a case there is a subsequence $y_{n_{j}}>b_{k-1}$ converging to $\lim w_{n}$ and such that $\lambda_{2}\left(y_{n_{j}-1}, \ldots, y_{n_{j}-m}\right)$ converges to a point $w \leq \lim w_{n}$.

Since

$$
y_{n_{j}} \leq \varphi\left(z_{k}, \lambda_{2}\left(y_{n_{j}-1}, \ldots, y_{n_{j}-m}\right)\right) \leq w_{n_{j}}
$$


one has

$$
\varphi\left(z_{k}, w\right)=\lim w_{n}>b_{k-1}=F\left(z_{k}\right)
$$

and then

$$
\varphi\left(z_{k}, w\right)-w>F\left(z_{k}\right)-w
$$

By applying (H2), we see that

$$
\lim w_{n}=\varphi\left(z_{k}, w\right)<w
$$

a contradiction.

Therefore, $\lim w_{n}=b_{k-1}$ and there exists $m_{k} \geq n_{k-1}$ such that

$$
y_{n}<b_{k-1}+\frac{1}{k} \quad \text { for all } n \geq m_{k}
$$

that is,

$$
F_{\varphi}\left(y_{n}\right) \geq a_{k} \quad \text { for all } n \geq m_{k} \text {. }
$$

Analogously, we see that there exists $n_{k} \geq m_{k}$ such that

$$
F_{\varphi}\left(y_{n}\right) \leq b_{k} \quad \text { for all } n \geq n_{k}
$$

\section{Proof of Lemma 2}

- Uniqueness of $F_{\varphi}$ : Let $y_{1}<y_{2}$ and $x$ in $[a, b]$ such that

$$
\frac{y_{i}-y}{\varphi(x, y)-y} \geq 1>0 \quad \text { for } i=1,2, y \in\left(y_{1}, y_{2}\right) .
$$

Then

$$
0<\varphi(x, y)-y<0
$$

a contradiction.

- (i): It suffices to prove the first assertion, because $[a, b]$ is a closed set and, by definition,

$$
\varphi(x, y)=\left\{\lim \sup \varphi\left(x_{n}, y_{n}\right): x_{n} \rightarrow x, y_{n} \rightarrow y, x_{n} \in(a, b), y_{n} \in(c, d)\right\}
$$

for all $(x, y) \in[a, b] \times[c, d]$. Assume now that $(x, y) \in(a, b) \times(a, b)$. We consider the following three possible situations. If $\varphi(x, y)=y$, it is obvious that $\varphi(x, y) \in(a, b)$.

On the other hand, if $\varphi(x, y)>y$ and $x^{\prime} \in(a, x)$, then

$$
a \leq y<\varphi(x, y)<\varphi\left(x^{\prime}, y\right) \leq F_{\varphi}\left(x^{\prime}\right) \leq b
$$

Finally, if $\varphi(x, y)<y$ and $x^{\prime} \in(x, b)$, then

$$
b \geq y>\varphi(x, y)>\varphi\left(x^{\prime}, y\right) \geq F_{\varphi}\left(x^{\prime}\right) \geq a .
$$


- (ii): Suppose $y \neq F_{\varphi}(x)$. Since $\varphi(x, y)=y \in \overline{\mathbb{R}}$, then $\varphi(x, y)-y=0$ or $\varphi(x, y)-y=\overline{\mathbb{R}}$. In any event, it cannot be the case that

$$
\frac{F_{\varphi}(x)-y}{\varphi(x, y)-y} \geq 1
$$

which contradicts hypothesis (H2), thus $y=F_{\varphi}(x)$.

- (iii): Since $F_{\varphi}([a, b]) \subset[a, b] \subset[c, d]$, it is worth considering the following three cases for each $x \in[a, b]$ : first, $x \in(a, b), F_{\varphi}(x) \in(c, d)$ and then (after probing continuity, monotonicity and statement (iv)), we proceed with the case $x \in\{a, b\}, F_{\varphi}(x) \in(c, d)$ and finally with $x \in[a, b], F_{\varphi}(x) \in\{c, d\}$.

Case $x \in(a, b)$ and $F_{\varphi}(x) \in(c, d)$ : Since $\varphi(x, y)>y$ when $y<F_{\varphi}(x)$ and $\varphi(x, y)<y$ when $y>F_{\varphi}(x)$, we see that

$$
\varphi\left(x, F_{\varphi}(x)\right)=F_{\varphi}(x)
$$

because of the continuity of $\varphi(x, \cdot)$.

- Monotonicity and (iv): Suppose

$$
F_{\varphi}\left(x_{1}\right) \leq F_{\varphi}\left(x_{2}\right) \quad \text { for } a \leq x_{1}<x_{2} \leq b .
$$

If $y \in\left[F_{\varphi}\left(x_{1}\right), F_{\varphi}\left(x_{2}\right)\right]$, then $y=a, y=b$ or $y \leq \varphi\left(x_{2}, y\right)<\varphi\left(x_{1}, y\right) \leq y$, thus

$$
\left[F_{\varphi}\left(x_{1}\right), F_{\varphi}\left(x_{2}\right)\right] \subset\{a, b\}
$$

- Continuity: If $x \in[a, b]$ and

$$
w \in I=\left(\liminf _{z \rightarrow x} F_{\varphi}(z), \limsup _{y \rightarrow x} F_{\varphi}(y)\right),
$$

then there exist two sequences $y_{n}, z_{n} \rightarrow x$ with

$$
F_{\varphi}\left(z_{n}\right)<w<F_{\varphi}\left(y_{n}\right)
$$

Thus,

$$
\varphi\left(z_{n}, w\right)<w<\varphi\left(y_{n}, w\right)
$$

and, by (ii), one has $\varphi(x, w)=w$. Since $w \in(c, d)$, this would imply $F_{\varphi}(x)=w$ for all $w \in I$, which is impossible.

- (iii) Case $x \in\{a, b\}$ and $F_{\varphi}(x) \in(c, d)$ : Since

$$
F_{\varphi}(t) \leq \varphi\left(t, F_{\varphi}(a)\right) \leq F_{\varphi}(a) \quad \text { for all } t \in(a, b)
$$

and because of the continuity of $F_{\varphi}$, we have

$$
F_{\varphi}(a)=\sup _{t} F_{\varphi}(t) \leq \sup _{t} \varphi\left(t, F_{\varphi}(a)\right) \leq F_{\varphi}(a)
$$


but

$$
\sup _{t} \varphi\left(t, F_{\varphi}(a)\right)=\varphi\left(a, F_{\varphi}(a)\right) .
$$

Analogously, it can be seen that $\varphi\left(b, F_{\varphi}(b)\right)=F_{\varphi}(b)$.

- (iii) Case $F_{\varphi}(x) \in\{c, d\}$ : First assume $F_{\varphi}(x)=c$ and recall that, by definition,

$$
\varphi(x, c)=\left\{\lim \sup \varphi\left(x_{n}, y_{n}\right): x_{n} \rightarrow x, y_{n} \rightarrow c, x_{n} \in(a, b), y_{n} \in(c, d)\right\} .
$$

Suppose $\varphi\left(x_{n}, y_{n}\right) \geq c^{\prime}>c$ for all $n$. Then

$$
1 \leq \frac{F_{\varphi}\left(x_{n}\right)-y_{n}}{\varphi\left(x_{n}, y_{n}\right)-y_{n}} \leq \frac{F_{\varphi}\left(x_{n}\right)-y_{n}}{c^{\prime}-y_{n}}
$$

which implies $F_{\varphi}\left(x_{n}\right) \geq c^{\prime}>c$, eventually for all $n$.

Since $F_{\varphi}\left(x_{n}\right) \rightarrow c$, we reach a contradiction. Therefore,

$$
\varphi\left(x, F_{\varphi}(x)\right)=\{c\}=\left\{F_{\varphi}(x)\right\} .
$$

Analogously, we see that $\varphi\left(x, F_{\varphi}(x)\right)=\{d\}$ when $F_{\varphi}(x)=d$.

\section{Examples and applications}

\subsection{The difference equation $y_{n}=A+\left(\frac{y_{n-k}}{y_{n-q}}\right)^{p}$ with $0<p<1$}

The paper [6] is devoted to prove that every positive solution to the difference equation

$$
y_{n}=A+\left(\frac{y_{n-k}}{y_{n-q}}\right)^{p}
$$

converges to the equilibrium $A+1$, whenever

$$
A \in(0,+\infty) \text { and } p \in(0, \min \{1,(A+1) / 2\}) \text {. }
$$

Here, $k, q \in\{1,2,3, \ldots\}$ are fixed numbers.

Although paper [6] complements [7], where the case $p=1$ had been considered, it should be noticed that the case $p=1, A \geq 1$ is not dealt with in [6]. Furthermore, we cannot assure the global attractivity in this case.

The results in [6] can be easily obtained by applying Theorem 1 above. Furthermore, we slightly improve the results in [6] by establishing the unconditional stability of the equilibrium $A+1$, whenever $A \in(0,+\infty), p \in(0, \min \{1,(A+1) / 2\})$. We may assume without loss of generality that the initial values $y_{1}, \ldots, y_{m}$ are greater than $A$. Here, and in the sequel $m=\max \{k, q\}$.

Let

$$
\begin{aligned}
& A \in(0,+\infty), \quad p \in(0,1), \quad a=c=A, \quad b=d=+\infty, \\
& \varphi(x, y)=A+\left(\frac{y}{x}\right)^{p},
\end{aligned}
$$


and

$$
\lambda\left(x_{1}, \ldots, x_{m}\right)=\left(x_{q}, x_{k}\right)
$$

Define $F_{\varphi}(+\infty)=A$, consider for the moment a fixed $x \in[A, \infty)$ and define $F_{\varphi}(x)$ to be the unique positive zero of the function $f_{x}$ given by

$$
f_{x}(y)=\varphi(x, y)-y \text {. }
$$

Notice that $f_{x}$ is concave, $f_{x}(0)=A>0$, and $f_{x}(+\infty)=-\infty$.

Clearly, $F_{\varphi}(x)$ is also the unique zero of the increasing function

$$
j_{x}(y)=\varphi(x, y)-F_{\varphi}(x) .
$$

Since

$$
j_{x}(A)=\frac{A^{p}-\left(F_{\varphi}(x)\right)^{p}}{x^{p}} \leq 0 \quad \text { and } \quad j_{x}(+\infty)=+\infty>0
$$

we see that condition (H2) holds and $\mu_{\varphi}=A+1$.

As for condition

$$
\operatorname{Fix}\left(F_{\varphi} \circ F_{\varphi}\right)=\operatorname{Fix}\left(F_{\varphi}\right)
$$

if $A \leq x<y<+\infty$ with

$$
A+\left(\frac{y}{x}\right)^{p}=y
$$

then $y>A+1$ and

$$
\varphi(y, x)-x=A+\left(\frac{x}{y}\right)^{p}-x=A+\frac{1}{y-A}-\frac{y}{(y-A)^{1 / p}} .
$$

Since the function

$$
h(z)=A+\frac{1}{z-A}-\frac{z}{(z-A)^{1 / p}}
$$

has a unique critical point in $(A,+\infty)$ and $h(A+1)=0, h(+\infty)=A$, the necessary and sufficient condition for (5) to hold is that $h^{\prime}(A+1) \geq 0$, that is, $p \leq(A+1) / 2$.

By this reasoning, we also get for free, unconditional stable convergence for several difference equations as, for instance:

$$
y_{n}=A+\left(\frac{y_{n-q} y_{n-r}}{y_{n-s} y_{n-t}}\right)^{p} \quad \text { with } 0<p<\min \{1 / 2,(A+1) / 4\}
$$

or

$$
y_{n}=A+\left(\frac{y_{n-q}+y_{n-r}}{y_{n-s}+y_{n-t}}\right)^{p} \quad \text { with } 0<p<\min \{1,(A+1) / 2\}
$$


just considering respectively

$$
\begin{aligned}
& \lambda\left(x_{1}, \ldots, x_{m}\right)=\left(\sqrt{x_{s} x_{t}}, \sqrt{x_{q} x_{r}}\right), \\
& \lambda\left(x_{1}, \ldots, x_{m}\right)=\left(\frac{x_{s}+x_{t}}{2}, \frac{x_{q}+x_{r}}{2}\right),
\end{aligned}
$$

where $m=\max \{q, r, s, t\}$.

4.2 The difference equation $y_{n}=\frac{\alpha+\beta y_{n-1}}{A+B y_{n-1}+C y_{n-2}}$

Here, $\alpha, \beta, A, B, C, y_{0}, y_{1} \geq 0$. In 2003, three conjectures on the above equation were posed in [8]. In all three cases $(B=0, \alpha, \beta, A, C>0 ; A=0, \alpha, \beta, B, C>0$; and $\alpha, \beta, A, B, C>0$, respectively) it was postulated the global asymptotic stability of the equilibrium. These conjectures have resulted in several papers since then (see [9-12]). Let us see when there is unconditional convergence.

Consider $a=c=0, b=c=+\infty$, and

$$
\varphi(x, y)=\frac{\alpha+\beta y}{A+D x}
$$

with $\alpha, \beta, A \geq 0, D>0$. We solve in $y$ the equation $y=\frac{\alpha+\beta y}{A+D x}$ to obtain

$$
y=\frac{\alpha}{A-\beta+D x},
$$

so we consider $A>\beta, \alpha>0$ to define

$$
F_{\varphi}(x)=\frac{\alpha}{A-\beta+D x} .
$$

A simple calculation shows that $(\mathrm{H} 2)$ holds, $\mu_{\varphi} \in(a, b)$ and $\operatorname{Fix}\left(F_{\varphi} \circ F_{\varphi}\right)=\operatorname{Fix}\left(F_{\varphi}\right)$ :

$$
\begin{aligned}
& \frac{F_{\varphi}(x)-y}{\varphi(x, y)-y}=1+\frac{\beta}{A-\beta+D x} \geq 1, \\
& \mu_{\varphi}=\frac{1}{2 D}\left(-A+\beta+\sqrt{\left((A-\beta)^{2}+4 D \alpha\right)}\right), \\
& \frac{F_{\varphi}\left(F_{\varphi}(x)\right)-x}{F_{\varphi}(x)-x}=\frac{(A-\beta+D x)(A-\beta)}{(A-\beta)^{2}+D x(A-\beta)+D \alpha}>0 .
\end{aligned}
$$

Therefore, $\mu_{\varphi}$ is an unconditional stable attractor of $\varphi$ in $(a, b)=(0,+\infty)$ whenever $A>$ $\beta \geq 0, D>0$ and $\alpha>0$.

If we choose

$$
\lambda\left(x_{1}, x_{2}\right)=\left(\frac{B x_{1}+C x_{2}}{B+C}, x_{1}\right)
$$

and $D=B+C$, we obtain unconditional stable convergence for the equation

$$
y_{n}=\frac{\alpha+\beta y_{n-1}}{A+B y_{n-1}+C y_{n-2}},
$$

whenever $A>\beta \geq 0, B+C>0, C \geq 0$ and $\alpha>0$. 
Other choices of $\lambda$ result on the unconditional stable convergence of difference equations such as

$$
y_{n}=\frac{\alpha+\beta y_{n-1}+\gamma y_{n-2}}{A+B y_{n-1}+C y_{n-2}}
$$

with $A>\beta+\gamma, B+C>0, \gamma \geq 0, \alpha>0, \beta \geq 0, C \geq 0$. Or

$$
y_{n}=\frac{\alpha+\beta \max \left\{y_{n-1}, y_{n-2}\right\}}{A+D \min \left\{y_{n-1}, y_{n-2}\right\}}
$$

with $A>\beta \geq 0, D>0, \alpha>0$.

\section{Competing interests}

The authors declare that they have no significant competing financial, professional, or personal interests that might have influenced the performance or presentation of the work described in this paper.

\section{Authors' contributions}

Both authors contributed to each part of this work equally and read and approved the final version of the manuscript.

\section{Acknowledgements}

Dedicated to Professor Jean Mawhin on the occasion of his 70th birthday.

We are grateful to the anonymous referees for their helpful comments and suggestions. This research was supported in part by the Spanish Ministry of Science and Innovation and FEDER, grant MTM2010-14837.

Received: 19 December 2012 Accepted: 20 March 2013 Published: 28 March 2013

\section{References}

1. Agarwal, RP: Difference Equations and Inequalities. Theory, Methods, and Applications, 2nd edn. Monographs and Textbooks in Pure and Applied Mathematics, vol. 228. Dekker, New York (2000)

2. El-Morshedy, HA, Gopalsamy, K: Oscillation and asymptotic behaviour of a class of higher-order non-linear difference equations. Ann. Mat. Pura Appl. 182, 143-159 (2003)

3. El-Morshedy, HA, Liz, E: Globally attracting fixed points in higher order discrete population models. J. Math. Biol. 53, 365-384 (2006)

4. Kocić, VL, Ladas, G: Global Behavior of Nonlinear Difference Equations of Higher Order with Applications. Mathematics and Its Applications, vol. 256. Kluwer Academic, Dordrecht (1993)

5. Beer, G: Topologies on Closed and Closed Convex Sets. Mathematics and Its Applications, vol. 268. Kluwer Academic, Dordrecht (1993)

6. Berenhaut, KS, Foley, JD, Stević, S: The global attractivity of the rational difference equation $y_{n}=A+\left(\frac{y_{n-k}}{y_{n-m}}\right)^{p}$. Proc. Am. Math. Soc. 136, 103-110 (2008)

7. Berenhaut, KS, Foley, JD, Stević, S: The global attractivity of the rational difference equation $y_{n}=1+\frac{y_{n-k}}{y_{n-m}}$. Proc. Am. Math. Soc. 135, 1133-1140 (2007)

8. Kulenović, MRS, Ladas, G, Martins, LF, Rodrigues, IW: The dynamics of $x_{n+1}=\frac{\alpha+\beta x_{n}}{A+B x_{n}+C x_{n-1}}$ : facts and conjectures. Comput. Math. Appl. 45, 1087-1099 (2003)

9. Merino, $\mathrm{O}$ : Global attractivity of the equilibrium of a difference equation: an elementary proof assisted by computer algebra system. J. Differ. Equ. Appl. 17, 33-41 (2011)

10. Su, Y-H, Li, W-T, Stević, S: Dynamics of a higher order nonlinear rational difference equation. J. Differ. Equ. Appl. 11, 133-150 (2005)

11. Su, Y-H, Li, W-T: Global asymptotic stability of a second-order nonlinear difference equation. Appl. Math. Comput. $168,981-989(2005)$

12. Su, Y-H, Li, W-T: Global attractivity of a higher order nonlinear difference equation. J. Differ. Equ. Appl. 11, 947-958 (2005)

doi:10.1186/1687-2770-2013-63

Cite this article as: Franco and Peran: Unconditional convergence of difference equations. Boundary Value Problems $20132013: 63$ 